Assume that $S-R_{1}{ }^{\prime}$ contains a point, say $U$, of $M_{2}$. Then by Condition 2 there exists an arc $U E_{3}$ contained in $M_{2}+E_{3}$. This is impossible for such an arc must contain a point of $J_{1}$. Therefore $M_{2}$ is contained in $R_{1}$. Similarly it can be proved that $M_{2}$ is contained in $S-R_{2}^{\prime}$ and so in $R_{3}$. Similarly it can be proved that $M_{2}$ is contained in $S-R_{3}^{\prime}$. Thus a contradiction is obtained. Therefore there do not exist three points of $L$.

Cornell University

\title{
TWO CLASSES OF PERIODIC ORBITS WITH REPELLING FORCES*
}

BY T. H. RAWLES

1. Introduction. The purpose of this paper is to obtain certain classes of periodic orbits $\nmid$ of a system consisting of one body of very great mass which attracts two mutually repellent bodies of very small mass. The forces of attraction and repulsion in the system are assumed to vary inversely as the squares of the distances. It will be shown that if certain conditions of symmetry are imposed the orbits of the two small bodies must lie either in two parallel planes or else be coplanar. In the former case they are circles about a line perpendicular to the two planes and passing through the large body. For the second type of orbit a development in powers of a parameter will be obtained.

2. The Equation of Motion. By a proper choice of the units of time and space we can make the coefficient of the intensity of the attraction equal to 1 . Then if $K^{2}$ is the

* Presented to the Society, February 25, 1928.

$\dagger$ The orbits referred to were first calculated by Langmuir, who employed numerical integration. (Physical Review, vol. 17 (1921), p. 339.) They have been discussed by Van Vleck in his monograph on Quantum Principles and Line Spectra. (Bulletin of the National Research Council, Number 54, p. 89.) 
ratio of the repulsion to the attraction the force function of the system is

$$
U=\frac{1}{r_{1}}+\frac{1}{r_{2}}-\frac{K^{2}}{\Delta^{\prime}}
$$

where $r_{1}$ and $r_{2}$ represent the distances between the two small bodies and the large body while $\Delta$ is the distance between the small bodies.

The unit of mass may be so chosen that the mass of each of the small bodies is unity. Then let $m$ denote the mass of the large body. The system will be referred to the rectangular axes $\xi, \eta, \xi$, and we shall indicate the coordinates of the large body by the subscript 0 , and the coordinates of the small bodies by the subscripts 1 and 2 . With this choice of units and coordinates, the equations of motion are

$$
\begin{aligned}
& m \ddot{\xi}_{0}=\frac{\partial U}{\partial \xi_{0}}, \quad m \ddot{\eta}_{0}=\frac{\partial U}{\partial \eta_{0}}, \quad m \ddot{\zeta}_{0}=\frac{\partial U}{\partial \zeta_{0}} ; \\
& \ddot{\xi}_{i}=\frac{\partial U}{\partial \xi_{i}}, \quad \ddot{\eta}_{i}=\frac{\partial U}{\partial \eta_{i}}, \quad \ddot{\zeta}_{i}=\frac{\partial U}{\partial \zeta_{i}}, \quad(i=1,2) .
\end{aligned}
$$

If $m$ is very large, the effect of the attraction of the other bodies on the large body is very small. We shall assume that this can be neglected altogether. In other words we assume that the mass of the large body is infinite as compared to that of the other two. Under this assumption we can neglect the right members of (2). These equations then show that the large body moves through space with uniform velocity in a straight line.

We now take this body as the origin, and denote the coordinates of the other two by $x_{i}, y_{i}, z_{i},(i=1,2$.) The equations of motion of the two small bodies then become

where

$$
\ddot{x}_{i}=\frac{\partial U}{\partial x_{i}}, \quad \ddot{y}_{i}=\frac{\partial U}{\partial y_{i}}, \quad \ddot{z}_{i}=\frac{\partial U}{\partial z_{i}},
$$

$$
U=\frac{1}{r_{1}}+\frac{1}{r_{2}}-\frac{K^{2}}{\Delta},
$$


and

$$
\left\{\begin{array}{l}
r_{i}{ }^{2}=x_{i}{ }^{2}+y_{i}{ }^{2}+z_{i}{ }^{2}, \\
\Delta^{2}=\left(x_{1}-x_{2}\right)^{2}+\left(y_{1}-y_{2}\right)^{2}+\left(z_{1}-z_{2}\right)^{2} .
\end{array}\right.
$$

3. Periodic Orbits. The periodic orbits with which we are concerned are those for which

$$
y_{1}-y_{2}=0, \quad z_{1}-z_{2}=0, \quad x_{1}-x_{2}=0 .
$$

With these restrictions equations (4) now become

$$
\left\{\begin{array}{l}
\ddot{x}_{1}=\frac{-x_{1}}{r_{1}^{3}}+\frac{k^{2}\left(x_{1}-x_{2}\right)}{\left(\left(x_{1}-x_{2}\right)^{2}\right)^{3 / 2}}=\frac{-x_{1}}{r_{1}^{3}}+\frac{k^{2}}{\left(2 x_{1}\right)^{2}}, \\
\ddot{y}_{1}=\frac{-y_{1}}{r_{1}^{3}}, \quad \ddot{z}_{1}=\frac{-z_{1}}{r_{1}^{3}},
\end{array}\right.
$$

with three similar equations for the coordinates of the other particle. The variables are now separated and we need only consider equations (8). When these are solved the motion of the other particle can be obtained from (7).

Let us now put $x_{1}=x, y_{1}=y, z_{1}=z, r_{1}=r$, and $k^{2} / 4=1-e^{2}$. Our equations may then be written in the form

$$
\ddot{x}=\frac{-x}{r^{3}}+\frac{1-e^{2}}{x^{2}}, \quad \ddot{y}=\frac{-y}{r^{3}}, \ddot{z}=\frac{-z}{r^{3}} .
$$

Two integrals of (9) can be found at once. They are

$$
\text { (10) } \frac{1}{2}\left(\dot{x}^{2}+\dot{y}^{2}+\dot{z}^{2}\right)=\frac{1}{r}-\frac{1-e^{2}}{x}+C, \quad \dot{z} y-z \dot{y}=h .
$$

Also we notice that if a solution

$$
x=X(e, t), \quad y=Y(e, t), \quad z=Z(e, t)
$$

is known, then another solution is

$$
x=a X\left(e, \frac{t-t_{0}}{a^{3 / 2}}\right), \quad y=a Y\left(e, \frac{t-t_{0}}{a^{3 / 2}}\right), z=a Z\left(e, \frac{t-t_{0}}{a^{3 / 2}}\right) .
$$


4. Periodic Solutions of Period $2 \pi$. We shall obtain solutions periodic with the period $2 \pi$. From the remark which we have just made we can infer the existence of solutions of any desired period. The existence of a solution developed in powers of $e$ as a parameter follows from a theorem of MacMillan.* When $e=0$, a solution of the equations is

$$
x=1, \quad y=0, \quad z=0 .
$$

The solutions which we shall proceed to develop are of the form

$$
\left\{\begin{array}{l}
x=1+e^{2} x_{2}+e^{4} x_{4}+\cdots, \\
y=e y_{1}+e^{3} y_{3}+\cdots, \\
z=e z_{1}+e^{3} z_{3}+\cdots .
\end{array}\right.
$$

When developed in powers of the parameter, the equations of motion are

$$
\left\{\begin{array}{c}
\sum_{0} e^{2 i} \ddot{x}_{2 i}=\left[\frac{3}{2}\left(y_{1}{ }^{2}+z_{1}^{2}\right)-1\right] e^{2}+\cdots \\
\sum_{0} e^{2 i+1} \ddot{y}_{2 i+1}=-y_{1} e+\left[-y_{3}\right. \\
\left.\quad+\frac{3}{2} y_{1}\left(2 x_{2}+y_{1}{ }^{2}+z_{1}^{2}\right)\right] e^{3}+\cdots \\
\sum_{0} e^{2 i+1} \ddot{z}_{2 i+1}=-z_{1} e+\left[-z_{3}\right. \\
\left.\quad+\frac{3}{2} z_{1}\left(2 x_{2}+y_{1}^{2}+z_{1}^{2}\right)\right] e^{3}+\cdots
\end{array}\right.
$$

Equating the coefficients of $e$ in the last two equations we obtain

$$
\ddot{y}_{1}+y_{1}=0, \quad \ddot{z}_{1}+z_{1}=0 .
$$

The solutions of these equations are

$$
\left\{\begin{array}{l}
y_{1}=c_{1} \cos t+c_{2} \sin t \\
z_{1}=c_{3} \cos t+c_{4} \sin t
\end{array}\right.
$$

where $c_{1}, \cdots, c_{4}$ are constants of integration. From the coefficients of $e$ in the first of (12), we have

* Transactions of this Society, vol. 13 (1912), p. 157. 


$$
\left\{\begin{aligned}
\ddot{x}_{2}= & \frac{3}{2}\left(y_{1}{ }^{2}+z_{1}^{2}\right)-1 \\
= & \frac{3}{2}\left[\left(\frac{c_{1}^{2}+c_{2}^{2}+c_{3}^{2}+c_{4}^{2}}{2}\right)\right. \\
& +\left(\frac{c_{1}^{2}+c_{3}^{2}-c_{2}^{2}-c_{4}^{2}}{2}\right) \cos 2 t \\
& \left.+\left(c_{1} c_{2}+c_{3} c_{4}\right) \sin 2 t\right]-1 .
\end{aligned}\right.
$$

Upon integration we obtain

$$
\begin{aligned}
x_{2}= & \left(\frac{3}{4}\left(c_{1}^{2}+c_{2}^{2}+c_{3}^{2}+c_{4}^{2}\right)-1\right) \frac{t^{2}}{2}+c_{5} t+c_{6} \\
& -\frac{3}{16}\left(c_{1}^{2}+c_{3}^{2}-c_{2}^{2}-c_{4}^{2}\right) \cos 2 t-\frac{3}{8}\left(c_{1} c_{2}+c_{3} c_{4}\right) \sin 2 t,
\end{aligned}
$$

$c_{5}$ and $c_{6}$ being arbitrary.

The periodicity conditions which we shall impose are that $x(2 \pi)-x(0)=0$ and $\dot{x}(2 \pi)-\dot{x}(0)=0$, with similar conditions for the other coordinates. Moreover, the coefficient of each power of the parameter must satisfy these conditions. When the periodicity conditions are applied to $x_{2}$ and $\dot{x}_{2}$ we see that

$$
\begin{aligned}
& \left(\frac{3}{4}\left(c_{1}^{2}+c_{2}^{2}+c_{3}^{2}+c_{4}^{2}\right)-1\right) \frac{(2 \pi)^{2}}{2}-2 \pi c_{5}=0, \\
& \left(\frac{3}{4}\left(c_{1}^{2}+c_{2}^{2}+c_{3}^{2}+c_{4}^{2}\right)-1\right)(2 \pi)=0 .
\end{aligned}
$$

From these relations we now find that

$$
c_{1}^{2}+c_{2}^{2}+c_{3}^{2}+c_{4}^{2}=4 / 3, \quad c_{5}=0 .
$$

Obviously the periodicity conditions imposed on $y_{1}, \dot{y}_{1}$, $z_{1}$ and $\dot{z}_{1}$ are satisfied identically.

When we proceed to the next approximation we have the equations 


$$
\left\{\begin{aligned}
\ddot{y}_{3}+y_{3} & =(3 / 2)\left(2 x_{2}+y_{1}^{2}+z_{1}^{2}\right) y_{1}, \\
\ddot{z}_{3}+z_{3} & =(3 / 2)\left(2 x_{2}+y_{1}^{2}+z_{1}^{2}\right) z_{1} .
\end{aligned}\right.
$$

These equations become

$$
\begin{aligned}
\ddot{y}_{3}+y_{3} & =\sum_{i} A_{i}^{(3)} \sin i t+\sum B_{i}^{(3)} \cos i t, \\
\ddot{z}_{3}+z_{3} & =\sum_{i} C_{i}^{(3)} \sin i t+\sum D_{i}^{(3)} \cos i t, \quad(i=1,3),
\end{aligned}
$$

where the coefficients of the periodic terms represent known functions of the constants of integration. Upon integrating the first of these two equations, we have

$$
\begin{gathered}
y_{3}=c_{1}^{(3)} \cos t+c_{2}^{(3)} \\
\sin t-A_{1}^{(3)} \frac{t \cos t}{2}+B_{1}^{(3)} \frac{t \sin t}{2} \\
+ \text { terms in } \cos 3 t \text { and } \sin 3 t
\end{gathered}
$$

where $c_{1}{ }^{(3)}$ and $c_{2}{ }^{(3)}$ are the new constants of integration. When we impose the condition that $y_{3}$ and $\dot{y}_{3}$ shall be periodic we find that $A_{1}^{(3)}=B_{1}^{(3)}=0$. In the same manner we can show that $C_{1}^{(3)}=D_{1}^{(3)}=0$.

5. Restrictions on the Constants. These four equations impose new restrictions on the constants $c_{i}$. They become

$$
\left\{\begin{array}{l}
B_{1}^{(3)}=3\left(c_{6}+\frac{1}{3}\right) c_{1}+c_{1} M+c_{2} N=0, \\
A_{1}^{(3)}=3\left(c_{6}+\frac{1}{3}\right) c_{2}-c_{2} M+c_{1} N=0, \\
D_{1}^{(3)}=3\left(c_{6}+\frac{1}{3}\right) c_{3}+c_{3} M+c_{4} N=0, \\
C_{1}{ }^{(3)}=3\left(c_{6}+\frac{1}{3}\right) c_{4}-c_{4} M+c_{3} N=0,
\end{array}\right.
$$

where

$$
M=\frac{3}{32}\left(c_{1}^{2}+c_{3}^{2}-c_{2}^{2}-c_{4}^{2}\right), \quad N=\frac{3}{16}\left(c_{1} c_{2}+c_{3} c_{4}\right) .
$$

When we multiply the four equations by $c_{4},-c_{3},-c_{2}, c_{1}$, respectively and add, the result is

$$
3\left(c_{6}+\frac{1}{3}\right)\left(c_{1} c_{4}-c_{2} c_{3}\right)=0 .
$$

There are three cases according as one factor is zero or both factors are zero. 
CASE I. $c_{6}+1 / 3=0, c_{1} c_{4}-c_{2} c_{3} \neq 0$.

The first of (16) shows that not all of the constants can be zero. It then follows from equations (18) that $M=N=0$. This gives us the two equations

$$
c_{1}^{2}+c_{3}^{2}-c_{2}^{2}-c_{4}^{2}=0, \quad c_{1} c_{2}+c_{3} c_{4}=0 .
$$

If, for example, $c_{2} \neq 0$ we have from the second of these two equations

$$
c_{1}^{2}=\frac{c_{3}^{2} c_{4}^{2}}{c_{2}^{2}} .
$$

When this is substituted in the first it becomes

$$
c_{3}^{2} c_{4}^{2}+c_{2}^{2} c_{3}^{2}-c_{2}^{4}-c_{4}^{2} c_{2}^{2}=0,
$$

or

$$
\left(c_{2}^{2}+c_{4}^{2}\right)\left(c_{3}^{2}-c_{2}^{2}\right)=0 .
$$

Since $c_{2} \neq 0$ we must have

$$
c_{3}=c_{2}, \quad c_{1}=-c_{4}, \quad \text { or } \quad c_{1}=c_{4}, \quad c_{3}=-c_{2} .
$$

Case I shows that as far as the approximation has been carried the motion is in a circle in a plane normal to the $x$ axis. In fact we have

$$
\begin{aligned}
y_{1}^{2}+z_{1}^{2} & =c_{1}^{2}+c_{2}^{2}=\frac{2}{3}, \\
x_{2} & =-\frac{1}{3} .
\end{aligned}
$$

CASE II. $c_{6}+1 / 3=0, c_{2} c_{3}-c_{1} c_{4}=0$.

It is easy to show that this case is impossible. We would have as in Case I $M=N=0$, and would obtain the same relations between the constants. When these relations are substituted in $c_{2} c_{3}-c_{1} c_{4}=0$ that equation becomes $c_{2}^{2}+c_{1}^{2}=0$, which is impossible since not all of these constants can be zero.

CASE III. $c_{6}+1 / 3 \neq 0, c_{2} c_{3}-c_{1} c_{4}=0$.

On multiplying the first of equations (14) by $c_{3}$, the second by $c_{1}$ and subtracting, since $c_{2} c_{3}-c_{1} c_{4}=0$, we obtain $c_{3} y_{1}$ $-c_{1} z_{1}=0$, which shows that the motion lies in a plane passing through the $x$ axis. (See Fig. 1.) 
6. The Orbits. We shall next show that when the process is continued each approximation gives rise to terms of the same type as those already found, and, therefore, that the periodic orbits are either circles about the $x$ axis, or else they lie entirely in a plane containing the $x$ axis.

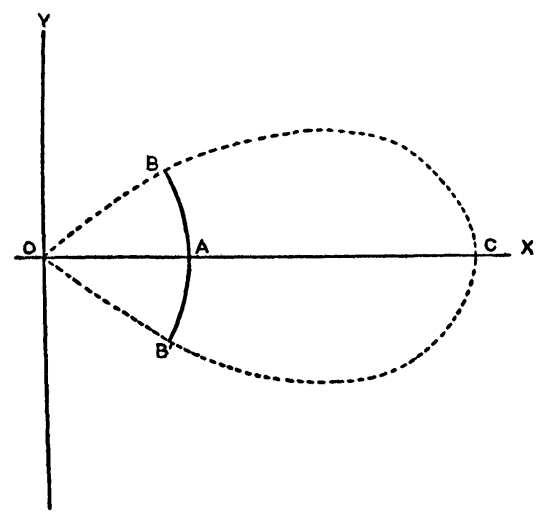

FIG. 1

The figure illustrates the path described by one of the particles for the case in which $e=1 / 2$. When $t=0$ it is at $A$. It reaches $B$ when $t=\pi / 2$, stops, and then returns along the same path to $A, t=\pi$. It then continues to $B^{\prime}, t=3 \pi / 2$, and finally completes the cycle by returning to $A$ when $t=2 \pi$. The position of the other particle is at all times symmetric to that of this one with respect to the $y$ axis. The curve of zero velocity (conchoid) is indicated by $O B C B^{\prime} O$.

For the discussion of Case I, let us suppose that when $t=0, y=0$. We then have

$$
c_{2}=c_{3}=\left(\frac{2}{3}\right)^{1 / 2}, \quad c_{4}=-c_{1}=0 .
$$

The solution, as far as we have carried it, is

$$
\begin{aligned}
& y=e\left(c_{2} \sin t\right)+e^{3}\left(c_{2}{ }^{(3)} \sin t\right), \\
& z=e\left(c_{2} \cos t\right)+e^{3}\left(c_{3}{ }^{(3)} \cos t+c_{4}{ }^{(3)} \sin t\right), \\
& x=1+e^{2}\left(-\frac{1}{3}\right) .
\end{aligned}
$$


The equation for $x_{4}$ becomes

$$
\begin{aligned}
\ddot{x}_{4}= & 3\left(y_{1} y_{3}+z_{1} z_{3}\right)+2 x_{2}+\frac{9}{2} x_{2}{ }^{2} \\
= & \left(\frac{3}{2}\left(c_{2} c_{2}{ }^{(3)}+c_{2} c_{3}^{\left({ }^{(3)}\right)}-\frac{1}{6}\right)\right. \\
& \quad+\frac{3}{2}\left\{c_{2}\left(c_{3}{ }^{(3)}-c_{2}{ }^{(3)}\right)\right\} \cos 2 t+\frac{3}{2} c_{2} c_{4}{ }^{(3)} \sin 2 t .
\end{aligned}
$$

Periodicity requires that

$$
c_{2}\left(c_{2}{ }^{(3)}+c_{3}^{(3)}\right)=1 / 9,
$$

and we have upon integration

$$
x_{4}=c_{6}{ }^{(4)}-\frac{3}{8} c_{2}\left(c_{3}^{(3)}-c_{2}^{(3)}\right) \cos 2 t-\frac{3}{8} 8 c_{2} c_{4}^{(3)} \sin 2 t .
$$

The equations for $y_{5}$ and $z_{5}$ now become

$$
\begin{aligned}
\ddot{y}_{5}+y_{5} & =\frac{3}{2} y_{1}\left(2 x_{4}+x_{2}^{2}+2\left(y_{1} y_{3}+z_{1} z_{3}\right)\right), \\
\ddot{z}_{5}+z_{5} & =\frac{3}{2} z_{1}\left(2 x_{4}+x_{2}^{2}+2\left(y_{1} y_{3}+z_{1} z_{3}\right)\right),
\end{aligned}
$$

which are when written out in full

$$
\begin{aligned}
& \ddot{y}_{5}+y_{5}=\frac{3}{2}\left(c_{2} \sin t\right)\left\{\left(\frac{2}{9}+2 c_{6}{ }^{(4)}\right)\right. \\
& \left.\quad+\frac{1}{4} c_{2}\left(c_{3}^{\left({ }^{(3)}-\right.}-c_{2}{ }^{(3)}\right) \cos 2 t+\frac{1}{4} c_{2} c_{4}{ }^{(3)} \sin 2 t\right\}, \\
& \ddot{z}_{5}+z_{5}=\frac{3}{2}\left(c_{2} \cos t\right)\left\{\left(\frac{2}{9}+2 c_{6}{ }^{(4)}\right)\right. \\
& \left.\quad+\frac{1}{4} c_{2}\left(c_{3}{ }^{(3)}-c_{2}{ }^{(8)}\right) \cos 2 t+\frac{1}{4} c_{2} c_{4}{ }^{(3)} \sin 2 t\right\} .
\end{aligned}
$$

When the products of the-trigonometric functions are expressed as functions of multiple angles it will be seen that for periodicity we must have

$$
c_{6}{ }^{(4)}=-1 / 9, \quad c_{3}{ }^{(3)}=c_{2}^{(3)}, \quad c_{4}^{(8)}=0 .
$$

The last of these conditions shows that $z_{3}$ contains no term in $\sin t$. The complementary function for $z_{5}$ may contain a term in $\sin t$ but the next integration will show that the coefficient of this term is zero.

The process can be continued indefinitely and our solution is of the form

$$
y=p_{1} \sin t, \quad z=p_{1} \cos t, \quad x=p_{2},
$$

where $p_{1}$ and $p_{2}$ are power series in $e$. The orbit is obviously a circle about the $x$ axis. 
We could have obtained this orbit by more elementary means. If we put

$$
\begin{aligned}
& x=\left(1-e^{2}\right)^{1 / 3}, \\
& y=\left(1-\left(1-e^{2}\right)^{2 / 3}\right)^{1 / 2} \sin \left(t-t_{0}\right), \\
& z=\left(1-\left(1-e^{2}\right)^{2 / 3}\right)^{1 / 2} \cos \left(t-t_{0}\right),
\end{aligned}
$$

it is easily seen that the equations of motion are satisfied.

For the discussion of the case where the motion, as far as the first approximation is concerned, lies in a plane containing the $x$ axis, let us choose that plane $z=0$. We shall take as the origin of time the instant when $y=0$. With this choice we have $c_{2}=(4 / 3)^{1 / 2}, c_{1}=c_{3}=c_{4}=0$.

Under these restrictions the equation for $z_{3}$ is $\ddot{z}_{3}+z_{3}=0$, and its solution is

$$
z_{3}=c_{3}^{(3)} \cos t+c_{4}^{(3)} \sin t .
$$

The constants of integration introduced in the complementary function of $x_{2}$ have already been determined by (16) and (18), and the equation for $z_{5}$ now becomes

$$
\begin{aligned}
\ddot{z}_{5}+z_{5}=z_{3}\left(3 x_{2}\right. & \left.+\frac{3}{2} y_{1}{ }^{2}\right) \\
& =-\frac{1}{4} c_{3}{ }^{(3)} \cos t-\frac{1}{8} c_{3}{ }^{(3)} \cos 3 t-\frac{1}{8} c_{4}{ }^{(8)} \sin 3 t .
\end{aligned}
$$

Now for a periodic solution it is obvious that $c_{3}{ }^{(3)}=0$. Therefore $z_{3}$ contains only a term in $\sin t$. If we continue this process imposing each time the condition that when $t=0, y=0$, we shall find that $z$ as well as $y$ contains only terms in $\sin (2 k+1) t$. This means of course that the particle crosses the $x$ axis when $t=n \pi$. The motion will always remain in the plane which contains the $x$ axis and the tangent to the orbit at the instant the particle crosses that axis because all the forces are acting in this plane. This can be easily verified by a consideration of the differential equations.

7. Indefinite Extension of the Process. It remains to show that the process of determining the constants can be carried on indefinitely. For this purpose we may take $z=0$ as the 
plane of motion and suppose that the following terms have been calculated:

$$
\left\{\begin{aligned}
x_{2 j} & =\sum_{0}^{j} a_{2 i}^{(2 j)} \cos 2 i t, \quad(j=0,1,2, \cdots, n) \\
y_{2 j+1} & =d_{2 j+1} \sin t+\sum_{1}^{j} a_{2 i+1}^{(2 j+1)} \sin (2 i+1) t
\end{aligned}\right.
$$

All the coefficients have been determined except $d_{2 n+1}$. When we equate the coefficients of $e^{2 n+2}$ in the equation for $x$ we find

$$
\ddot{x}_{2 n+2}=x_{2 n+2}(-1+3-2)+3 y_{1} y_{2 n+1}+\text { known terms. }
$$

This may be written in the form

$$
\ddot{x}_{2 n+2}=\frac{3}{2} c_{2} d_{2 n+1}(1-\cos 2 t)+\sum_{0}^{n+1} A_{2 i}^{(2 n+2)} \cos 2 i t,
$$

where all the coefficients are known except $d_{2 n+1}$. The arguments of the periodic functions must be even multiples of the time for they all enter from $x$ or $y^{2}$ which contain only terms with arguments of that form.

The integration of this equation will produce a secular term unless

$$
\frac{3}{2} c_{2} d_{2 n+1}+A_{0}^{(2 n+2)}=0 .
$$

Having imposed this condition, we find that

$$
x_{2 n+2}=b_{2 n+2} t+d_{2 n+2}+\sum_{1}^{n+1} a_{2 i}^{(2 n+2)} \cos 2 i t,
$$

where $b_{2 n+2}$ and $d_{2 n+2}$ are constants of integration and

$$
a_{2 i}^{(2 n+2)}=\frac{-A_{2 i}^{(2 n+2)}}{(2 i)^{2}} .
$$

For periodicity we must of course put

$$
b_{2 n+2}=0 \text {. }
$$


We now determine $y_{2 n+3}$ by equating the coefficients of $e^{2 n+3}$ in the $y$ equation. This gives

$$
\ddot{y}_{2 n+3}+y_{2 n+3}=3 y_{1} x_{2 n+2}+\text { known terms, }
$$

which may be written in the form

$$
\ddot{y}_{2 n+3}+y_{2 n+3}=3 c_{2} d_{2 n+2} \sin t+\sum_{0}^{n+1} A_{2 i+1}^{(2 n+3)} \sin (2 i+1) t .
$$

The arguments of the periodic functions are odd multiples of the time because they arise from terms such as $y^{2 k+1}$ and $y^{2 k+1} x^{2 h}$. If the coefficient of $\sin t$ in the right member is not zero a secular term will be introduced. This requires that

$$
3 c_{2} d_{2 n+2}+A_{1}{ }^{2 n+3}=0 .
$$

When the equation is integrated we have

(24) $y_{2 n+3}=d_{2 n+3} \sin t+b_{2 n+3} \cos t+\sum_{1}^{n+1} a_{2 i+1}^{(2 n+3)} \sin (2 i+1) t$, where

$$
a_{2 i+1}^{(2 n+3)}=\frac{A_{2 i+1}^{(2 n+3)}}{1-(2 i+1)^{2}} .
$$

We may suppose that the origin of time has been fixed by the condition $y=0$ when $t=0$. This determines the remaining constant and we have

$$
b_{2 n+3}=0 .
$$

We now have determined all the coefficients to and including $x_{2 n+2}$ and $y_{2 n+2}$, except the coefficient of $\sin t$ in the latter. Equations (22) and (24) are of the same form as (20), with $n$ replaced by $n+1$ and it is evident that the process can be carried on indefinitely.

8. Development of $x$ and $y$ in Terms of the Parameter. The development of $x$ and $y$ has been carried as far as terms involving the fifth power of the parameter. The expressions found are 


$$
\left\{\begin{aligned}
& x=1+\left(-\frac{3}{8}+\frac{1}{4} \cos 2 t\right) e^{2} \\
&+\left(-\frac{391}{4608}+\frac{29}{384} \cos 2 t-\frac{5}{1536} \cos 4 t\right) e^{4} \\
& y=(3)^{1 / 2}\left[\left(\frac{2}{3} \sin t\right) e+\left(\frac{1}{96} \sin 3 t\right) e^{3}\right. \\
&+\left(-\frac{3307}{331776} \sin t+\frac{91}{36864} \sin 3 t\right. \\
&\left.\left.+\frac{37}{110592} \sin 5 t\right) e^{5}\right]
\end{aligned}\right.
$$

For the case of the helium atom $k^{2}=1 / 2$ and the parameter $e$ must be given the value $(7 / 8)^{1 / 2}$. For this value of the parameter the convergence is not rapid, and the terms which we have calculated do not give the same degree of accuracy as the numerical integration of Langmuir. However the two results are in sufficient agreement to verify each other.

The numerical results are easily checked by means of the energy integral

$$
\frac{1}{2}\left(\dot{x}^{2}+\dot{y}^{2}\right)=\frac{1}{r}-\frac{1-e^{2}}{x}+C .
$$

The constant of integration is itself a power series in the parameter. As far as the sixth power of the parameter, it is

$$
C=-\frac{1}{3} e^{2}+\frac{1}{16} e^{4}+\frac{127}{10368} e^{6} .
$$

YALE UNIVERSITY 